\title{
Tuberculous Spondylodiscitis after Lumbar Microdiscectomy
}

\author{
Ozkan Ozger ${ }^{1}$ and Necati Kaplan² \\ ${ }^{1}$ Neurosurgery Clinic, Istanbul Rumeli University, Medicalpark Canakkale Hospital, Istanbul, Turkey \\ ${ }^{2}$ Neurosurgery Clinic, Istanbul Rumeli University, Corlu Reyap Hospital, Istanbul, Turkey
}

\begin{abstract}
Postoperative spondylodiscitis (PSD) and postoperative osteomyelitis (POM) are known complications of lumbar disc surgery. Many infectious agents play a role in its etiology and it is mostly bacterial.

A 55-year male patient underwent lumbar microdiscectomy (LMD) for left L4-5 disc hernia. Lumbar magnetic resonance images of the patient in the postoperative eighth week showed an infection, thought to be due to tuberculosis (TB) in the operation site and adjacent vertebrae. The patient who was positive for the QuantiFERON-TB Gold In-Tube (QFT-GIT) test was diagnosed with TB-induced PSD. The patient received anti-TB treatment consisting of ethambutol, isoniazid, pyrazinamide, and rifampin.

We report a very rare case of PSD due to TB infection after LMD. Clinical results and management of the patient was compared with other patients with similar characteristics in the literature.
\end{abstract}

Key Words: Discectomy, Osteomyelitis, Spondylodiscitis, Tuberculosis.

How to cite this article: Ozger O, Kaplan N. Tuberculous Spondylodiscitis after Lumbar Microdiscectomy. J Coll Physicians Surg Pak 2020; 30(09):983-986.

\section{INTRODUCTION}

The spinal region is the most common site of tuberculosis (TB) infection other than lungs. ${ }^{1}$ Postoperative spondylodiscitis (PSD) and postoperative osteomyelitis (POM) are known complications of lumbar disc surgery. There are many causes of PSD and POM, TB being one of them. The presence of immunodeficiency increases the risk of TB infection. ${ }^{2}$ Mycobacteria reach the surgical site after surgery by two mechanisms. The first one is the hematogenous spread of active pulmonary TB from the lung to the surgical site in the spinal region. The second one is the local reactivation of an inactive TB focus in the body. ${ }^{1-4}$

Herein, we present a very rare case of TB in a middle-aged man, who underwent lumbar microdiscectomy (LMD) for left L4-5 lumbar disc hernia (LDH), developed PSD and POM after LMD treatment.

A55-year male patient was admitted to the neurosurgery outpatient clinic with complaints of low back pain and weakness in his left leg for the last two weeks. Visual analog scale (VAS) score was 5 for the low back pain and 9 for leg pain.

Correspondence to: Dr. Ozkan Ozger, Neurosurgery Clinic, Istanbul Rumeli University, Medicalpark Canakkale Hospital, Istanbul, Turkey

E-mail: ozkanozger@hotmail.com

Received: January 31, 2020; Revised: February 07, 2020;

Accepted: February 07, 2020

DOI: https://doi.org/10.29271/jcpsp.2020.09.983

\section{CASE REPORT}

There was no history of TB or other immunodeficiency disease in his medical history. He was a tailor by profession. There was no fever or external finding on the body in his physical examination. Neurological examination revealed loss of strength in the left extensor hallucis longus muscle $2 / 5$ and in the anterior muscle of the tibialis $1 / 5$. He had hypoesthesia in the left L5 dermatome. The Lasègue test on the left was positive at 30 degrees. There was no anal sphincter disorder or urinary incontinence. Other systematic examination results were normal.

Lumbar magnetic resonance imaging (MRI) was taken for diagnosis. Disc herniation and root compression were detected at the left L4-5 (Figure 1). There were no suspicious signs of infection in the intervertebral disc (IVD) and adjacent endplates or vertebral bodies. Preoperative white blood cell (WBC), one-hour erythrocyte sedimentation rate (ESR) and C-reactive protein (CRP) values and reference ranges were $10.27 \times 10^{3} / \mathrm{mm}^{3}$ $\left(3.98-10.2 \times 10^{3} / \mathrm{mm}^{3}\right), 6 \mathrm{~mm} /$ hour $(0-20 \mathrm{~mm} /$ hour $)$ and 0.79 $\mathrm{mg} / \mathrm{L}(0-5 \mathrm{mg} / \mathrm{L})$, respectively. The patient underwent $\mathrm{L} 4-5$ microdiscectomy surgery. During the microdiscectomy operation, the extruded disc material causing left foraminal compression was observed, it was extracted, and the tissues were not found to be infective. Disc material was not sent for culture because the patient had no symptoms due to spondylodiscitis or infection at the first presentation. It was observed that the patient's power loss recovered almost completely in the early postoperative period. The patient, whose active complaints had passed, was discharged on the 4th day of operation. Ten days after discharge, the patient was checked in the outpatient clinic, 
and his neurological examination showed that the bilateral Lasègue test was negative, muscle strength was complete, the wound was clean, and there were no active complaints.

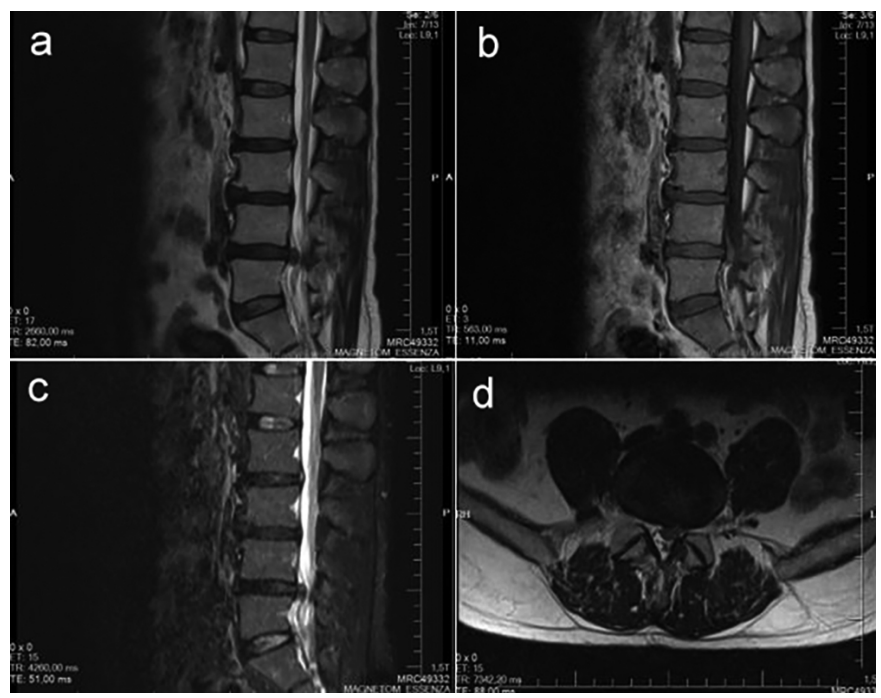

Figure 1: Preoperative lumbar MRI scan demonstrates left L4-5 disc herniation. (a) Sagittal T2-weighted MRI; (b) Sagittal T1-weighted MRI; (c) Sagittal T2 Short-Tau Inversion-Recovery (T2-STIR) MRI; (d) Axial T2weighted MRI.

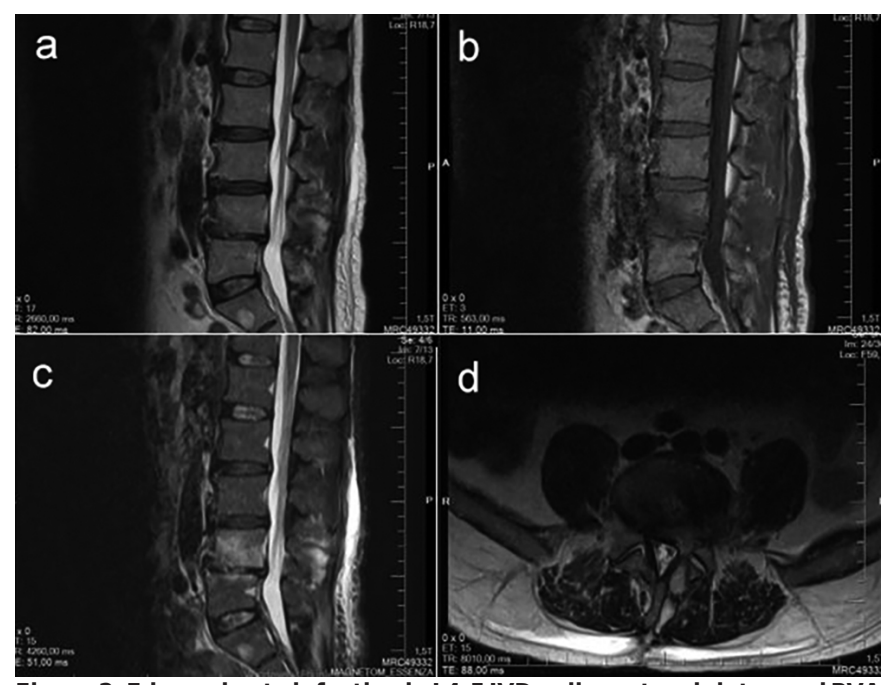

Figure 2: Edema due to infection in L4-5 IVD, adjacent endplates and PVA on control lumbar MRI taken at 8th postoperative week. (a) Sagittal T2weighted MRI; (b) Sagittal T1-weighted MRI; (c) SagittaI T2-STIR MRI; (d) AxialT2-weighted MRI.

He presented again with severe low back and left leg pain, sweating and fever in the sixth postoperative week. He could not move due to low back pain. Although the patient described mechanical low back pain, he also described radicular findings. It was understood that the pain in the anamnesis of the patient increased with movement, decreased at rest, and there were no night sweating and nocturnal pain. It was understood that his pain increased only when he moved in bed. The VAS score was 10 for low back pain and 5 for the leg pain. In the neurological examination of the patient, the Lasègue test on the left was positive at 30 degrees, there was valleix point sensitivity on the left, and paraspinal sensitivity was present. He did not describe hypoesthesia and had no anal and urinary sphincter defects.
There was no muscle strength deficit. The WBC, ESR, and CRP values were $7.67 \times 10^{3} / \mathrm{mm}^{3}, 116 \mathrm{~mm} /$ hour and $55.8 \mathrm{mg} / \mathrm{L}$, respectively. Control MRI taken at the postoperative eighth week showed an edematous appearance due to infection in L4-5 IVD, adjacent endplates, and paravertebral area (PVA) (Figure 2). Thoracic computed tomography (CT) images revealed a consolidated area with irregular contours and infiltrative appearance at the right lung apical posterior segment. Biopsy from the surgical site and a surgical procedure of antibiotic washing were recommended to the patient but he refused. There was no growth in TB cultures of blood and saliva samples. Only QuantiFERON-TB Gold In-Tube (QFT-GIT) test was positive. Anti-TB treatment consisting of ethambutol, isoniazid, pyrazinamide, and rifampin was initiated.

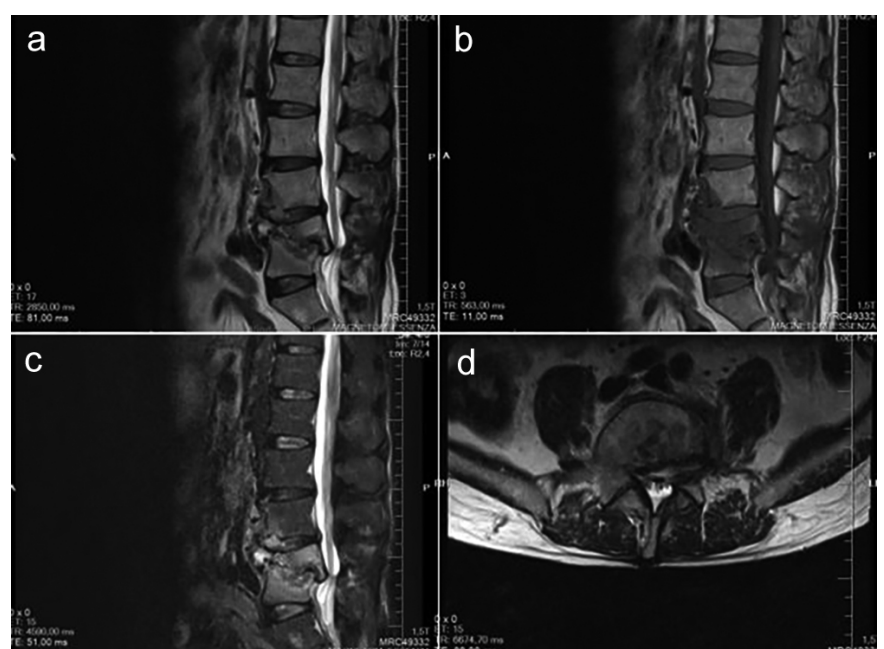

Figure 3: Progressive destruction, collapse and invasion of the spinal canal in the L4 and L5 vertebral bodies on control lumbar MRI taken at 6 months postoperatively. (a) Sagittal T2-weighted MRI; (b) Sagittal T1weighted MRI; (c) Sagittal T2-STIR MRI; (d) AxialT2-weighted MRI.

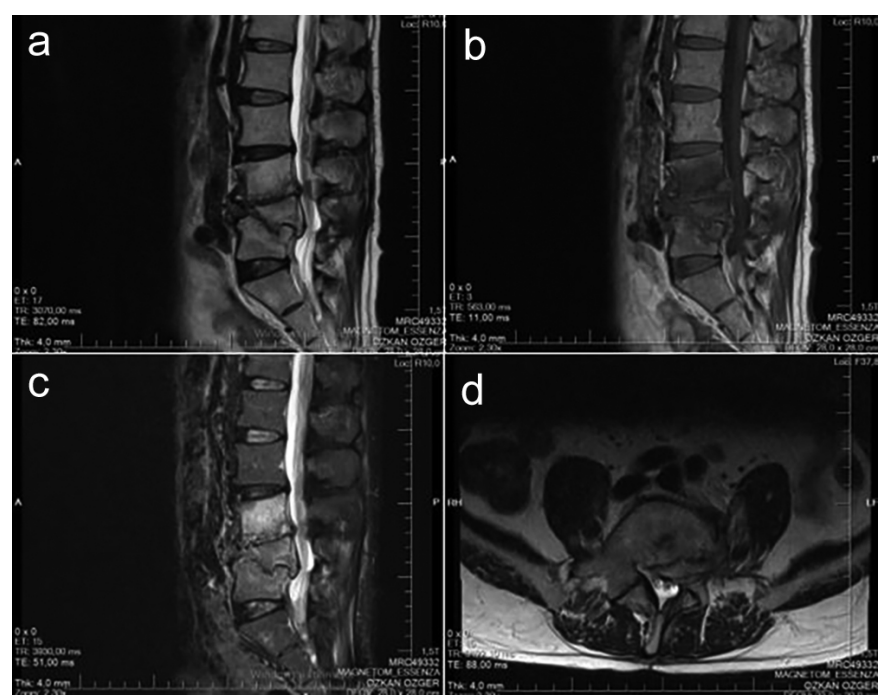

Figure 4: In postoperative 9th month control lumbar MRI, PVA, L4-5 IVD, L4 and L5 vertebral corpus edema decreased while $L 3$ vertebral bodies edema increased. (a) Sagittal T2-weighted MRI; (b) Sagittal T1-weighted MRI; (c) SagittaIT2-STIR MRI; (d) AxialT2-weighted MRI.

The WBC, ESR, and CRP values at $16^{\text {th }}$ postoperative week were $10.57 \times 10^{3} / \mathrm{mm}^{3}, 39 \mathrm{~mm} /$ hour and $3.71 \mathrm{mg} / \mathrm{L}$, respectively. In lumbar MRI taken at sixth postoperative month, the infection-in- 
duced edematous appearance in PVA was decreased, but edema had spread to the anterior part of the $L 3$ vertebra in addition to L4-5 IVD and adjacent endplates. Furthermore, progressive destruction, collapse, and invasion into the spinal canal were detected in the L5 vertebral body, and particularly in the L4 vertebral body (Figure 3). However, the patient's clinical condition was better. The VAS score was 5 for the low back pain and 4 for the leg pain. In the lumbar MRI taken at ninth postoperative month, edema was decreased in PVA, L4-5 IVD, L4, and L5 vertebral bodies; whereas, it increased in the L3 vertebral body (Figure 4). The VAS score was 4 for the low back pain and 3 for the leg pain. Control lumbar MRI images taken at the first postoperative year showed decreased infection-related edematous appearance in all vertebral bodies, PVA and IVD regions (Figure 5). The VAS score was 1 for the low back pain and 2 for the leg pain. After the laboratory and imaging results returned to normal, the patient returned to work at first year of the treatment. In the postoperative second year, the VAS score was 0 for the low back pain and 1 for the leg pain. There were no signs or complaints exceptfor mild weakness in the left foot at follow-up two years and eight months aftersurgery.

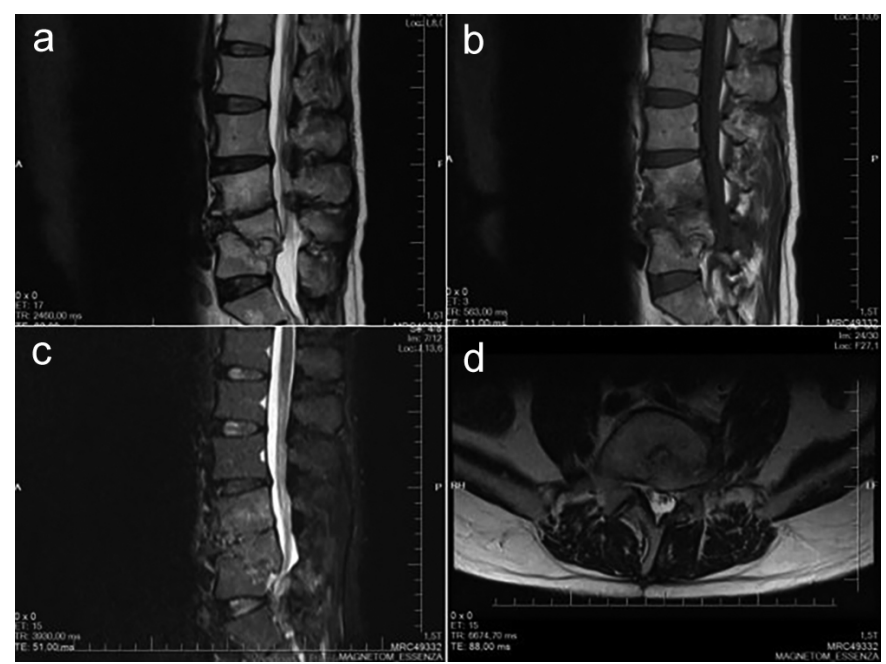

Figure 5: On postoperative 1st year control lumbar MRI, all vertebral bodies, PVA and IVD regions had decreased edematous appearance due to infection. (a) Sagittal T2-weighted MRI; (b) Sagittal T1-weighted MRI; (c) SagittalT2-STIR MRI; (d) AxialT2-weighted MRI.

\section{DISCUSSION}

The first case of TB-induced PSD was described in a 51-year-old woman undergoing LMD in 2009. Microdiscectomy was performed to the right L4-5 and upon the appearance of signs and symptoms in the early period, the patient was diagnosed with Pott's disease. It has been reported to cause misdiagnosis by mimicking the pyogenic causes of PSD. ${ }^{2}$

One of the most important causes of failed back surgery is PSD. The most common bacterial agents are staphylococci and streptococci. In a case report published in 2010, a 35-year female patient underwent L2-3 discectomy. She presented to the Emergency Department with signs of infection in the 16th postoperative week. Tubercular pathology was detected in the biopsy taken from the surgical site with $\mathrm{CT}$ and anti-TB treatment was initiated. $^{5}$
TB-induced spondylitis may also occur after percutaneous vertebroplasty (PVP) and percutaneous kyphoplasty (PKP). ${ }^{6-8}$ Compared to LDH, vertebral collapse fractures are more likely to be misdiagnosed preoperatively due to the presence of preoperative vertebral edema. ${ }^{9}$

In a case report of a 59-year female patient, a sample was collected from the surgical site. The diagnosis was made two days after surgery by demonstrating acid fast bacilli (AFB) on smear examination by Infectious Dieseases Department, and chronic granulomatous inflammation was confirmed on histopathology. ${ }^{10}$

In a study, the sensitivity and specificity of the QFT-GIT test in the diagnosis of active TB has been reported to be $84.8 \%$ and $60.5 \%$, respectively. ${ }^{4}$ Therefore, if the QFT-GIT test is positive, treatment can be initiated to prevent the loss of time.

As can be understood from the above discussion, TB-induced PSD and POM may develop after minimally invasive spinal surgeries such as microdiscectomy, PVP and PKP. Symptoms related to TB infection may occur after weeks and months or after years. Fatal consequences or disability may sometimes occur due to delay in diagnosis.

This patient is the sixth case in the literature, who developed PSD and POM after lumbar discectomy due to LDH. Unlike other cases, this patient refused biopsy and second surgical intervention options. Therefore, the patient was diagnosed and treated using QTB-GIT test, by which results can be obtained within 24 hours. It is recommend to use this test to prevent delays in the diagnosis and treatment of patients suspected of TB-induced PSD and POM.

\section{PATIENT'S CONSENT:}

Informed consent was taken from the patient's parents for publication of this case report and related images.

\section{CONFLICT OF INTEREST:}

The authors declared no conflict of interest.

\section{AUTHORS' CONTRIBUTION:}

ÖÖ: Interpretation, operating surgeon, data acquisition, literature review, manuscript drafting, supervision, critical revision, finalapproval.

NK: Interpretation, conception and design, manuscript review, editing, critical review, final approval.

\section{REFERENCES}

1. Celik SE. Pott disease mimics postsurgical pyogenic spondylodiscitis. J Neurosurg Sci 2009; 53(4):161-4.

2. de Araujo AO, Soares TQ, Torelli AG, Ono AHA, Marcon RM, Cristante AF, et al. Complicated lumbar tuberculous spondylodiscitis in disseminated tuberculosis, treated using a non-conventional anterior support system for hydrostatic distraction: A case report. Acta Ortop Bras 2018; 26(6):401-5. doi: 10.1590/1413-785220182606178095.

3. Du F, Xie L, Zhang Y, Gao F, Zhang H, Chen W, et al. Prospective comparison of QFT-GIT and T-SPOT.TB assays for diagnosis of active tuberculosis. Sci Rep 2018; 
8(1):5882. doi: 10.1038/s41598-018-24285-3.

4. Dulani R, Shrivastava S, Singh P. A rare case report: Tubercular spondylodiscitis following lumbar disc surgery. Asian Pac J Trop Med 2010; 3(6):496-8. doi.org/10.1016/ S1995-7645 (10)60120-4.

5. Ivo R, Sobottke R, Seifert H, Ortmann M, Eysel P. Tuberculous spondylitis and paravertebral abscess formation after kyphoplasty: A case report. Spine (Phila Pa 1976) 2010; 35(12):E559-63. doi: 10.1097/BRS. 0b013e3181celaab.

6. Kang JH, Kim HS, Kim SW. Tuberculous spondylitis after percutaneous vertebroplasty: misdiagnosis or complication? Korean J Spine 2013; 10(2):97-100. doi: 10.14245/kjs.2013.10.2.97.

7. Kim HJ, Shin DA, Cho KG, Chung SS. Late onset tuberculous spondylitis following kyphoplasty: a case report and review of the literature. Korean J Spine 2012; 9(1):28-31. doi: 10.14245/kjs.2012.9.1.28.

8. Lai PJ, Liao JC, Chen LH, Lai PL. Tuberculous spondylitis after percutaneous vertebroplasty: A case series of 9 cases. Biomed J 2019; 42(4):285-92. doi: 10.1016/j.bj. 2019.04.002.

9. Lotfinia I, Vahedi P. Late-onset post-diskectomy tuberculosis at the same operated lumbar level: case report and review of literature. Eur Spine J 2010; 19(Suppl 2):S226-32. doi: 10.1007/s00586-010-1420-z.

10. Özdoğan S, Yaltırık CK, Düzkalır AH, Demirel N, Kaya M, Atalay B. Spinal tuberculosis mimicking failed back surgery. Am J Case Rep 2018; 19:249-53. doi: 10.12659/ajcr. 907409. 